\title{
On a strange evaluation of the hypergeometric series by Gosper. II
}

\author{
Akihito Ebisu
}

October 15, 2018

\begin{abstract}
There are many identities for the hypergeometric series presented in the article "Special values of the hypergeometric series" by Ebisu. In this note, we obtain a new hypergeometric identity, which includes some of these identities as special cases. We notice that this identity closely relates to a strange evaluation by Gosper.

Key Words and Phrases: the hypergeometric series, hypergeometric identity.

2010 Mathematics Subject Classification Numbers: Primary 33C05
\end{abstract}

\section{Introduction and Main Theorem}

We begin with the binomial theorem,

$$
{ }_{1} F_{0}\left(\begin{array}{l}
a \\
-
\end{array} ; x\right):=\sum_{n=0}^{\infty} \frac{(a)_{n}}{n !} x^{n}=(1-x)^{-a},
$$

where $(a)_{n}$ is the Pochhammer symbol defined by

$$
(a)_{n}:=\frac{\Gamma(a+n)}{\Gamma(a)}= \begin{cases}1 & n=0 \\ a(a+1) \cdots(a+n-1) & n \in \mathbb{N} .\end{cases}
$$

Let us consider a generalization of ${ }_{1} F_{0}(a ;-; x)$ defined as

$$
{ }_{2} F_{1}\left(\begin{array}{c}
a, b \\
c
\end{array} ; x\right):=\sum_{n=0}^{\infty} \frac{(a)_{n}(b)_{n}}{(c)_{n} n !} x^{n}
$$

where $c \notin \mathbb{Z}_{\leq 0}$. This series is called the hypergeometric series. Unfortunately, in the general case, with unrestricted values of $(a, b, c, x)$, the hypergeometric series ${ }_{2} F_{1}(a, b ; c ; x)$ cannot be expressed in terms of well-known functions, or to be more exact, gamma functions together with elementary functions (see [10]). However, ${ }_{2} F_{1}(a, b ; c ; x)$ can be evaluated for parameter values satisfying certain conditions. For instance,

$$
{ }_{2} F_{1}\left({ }_{c}^{a, b} ; 1\right)=\frac{\Gamma(c) \Gamma(c-a-b)}{\Gamma(c-a) \Gamma(c-b)}
$$


holds for $\Re(c-a-b)>0$. There are many other known identities for ${ }_{2} F_{1}(a, b ; c ; x)$. Most of these identities have been derived using Gosper's algorithm, the W-Z method, Zeilberger's algorithm (see [8] and [9]), and the method of contiguity relations, which was recently introduced in [2]. In [2], a number of identities for ${ }_{2} F_{1}(a, b ; c ; x)$ are tabulated (see also [3], [5] and [6]).

First, we expand the definition of ${ }_{2} F_{1}(a, b ; c ; x)$. Even if the parameter $c$ is a non-positive integer, we define ${ }_{2} F_{1}(a, b ; c ; x)$ as follows if $b$ is a non-positive integer satisfying $c<b$ :

$$
{ }_{2} F_{1}\left(\begin{array}{c}
a, b \\
c
\end{array} ; x\right):=\sum_{n=0}^{|b|} \frac{(a)_{n}(b)_{n}}{(c)_{n} n !} x^{n} \quad\left(b, c \in \mathbb{Z}_{\leq 0} ; c<b\right) .
$$

With this expanded definition, for example, the following identities hold for any $m \in \mathbb{Z}_{\geq 0}$ :

$$
\begin{aligned}
& { }_{2} F_{1}\left(\begin{array}{cc}
\left.a, 3 a+1 ; \frac{3}{2}\right) \\
3 a & \text { if } a=-1-m, \\
\frac{(-3)^{3 m}(1 / 3)_{m}(5 / 3)_{2 m}}{2^{3 m}(2)_{3 m}} & \text { if } a=-1 / 3-m, \\
\frac{(-3)^{3 m}(2 / 3)_{m}(7 / 3)_{2 m}}{2^{3 m+1}(3)_{3 m}} & \text { if } a=-2 / 3-m,
\end{array}\right. \\
& { }_{2} F_{1}\left(\begin{array}{cc}
a, 4 a+1 \\
4 a
\end{array} ; \frac{4}{3}\right)= \begin{cases}\frac{(-1)^{m} 2^{8 m}(1 / 4)_{m}(7 / 4)_{3 m}}{3^{4 m}(2)_{4 m}} & \text { if } a=-1-m, \\
\frac{(-1)^{m} 2^{8 m+1}(1 / 2)_{m}(5 / 2)_{3 m}}{3^{4 m+1}(3)_{4 m}} & \text { if } a=-1 / 2-m, \\
\frac{5(-1)^{m} 2^{8 m-1}(3 / 4)_{m}(13 / 4)_{3 m}}{3^{4 m+2}(4)_{4 m}} & \text { if } a=-3 / 4-m .\end{cases}
\end{aligned}
$$

The formulae (1.1) and (1.2) appear as $(1,3,3-3)(\mathrm{i})$ and $(1,4,4-1)(\mathrm{i})$, respectively, in [2]. These formulae are treated individually in [2]. However, looking closely at them, we realize that they have a similarity: Their left-hand sides both have the form

$$
{ }_{2} F_{1}\left(\begin{array}{c}
\alpha, 1-k \\
-k
\end{array} ; \frac{k}{\alpha+k}\right),
$$

where $k \in \mathbb{N}$. Hence, if we are able to evaluate (1.3), then 1.1 and $(1.2)$ follow as special cases.

From Gosper's algorithm, we find that

$$
\frac{(\alpha)_{n}(1-k)_{n}}{(-k)_{n} n !}\left(\frac{k}{\alpha+k}\right)^{n}=f(n+1)-f(n)
$$

where

$$
f(n):=\frac{\alpha+k}{k} \cdot \frac{(\alpha+1)_{n-1}}{(n-1) !}\left(\frac{k}{\alpha+k}\right)^{n}
$$


Formula (1.4) implies

$$
{ }_{2} F_{1}\left(\begin{array}{c}
\alpha, 1-k \\
-k
\end{array} ; \frac{k}{\alpha+k}\right)=\frac{(\alpha+1)_{k}}{k !}\left(\frac{k}{\alpha+k}\right)^{k},
$$

and, from this, we have the following theorem:

Theorem 1. For any $(\alpha, k)$ satisfying

$$
(\alpha, k) \in\{(\alpha, k) \in \mathbb{C} \times \mathbb{N} \mid \alpha+k \neq 0\},
$$

we have (1.5). The identity (1.5) includes the formulae (1.1) and (1.2), and also the formulae $(1,5,5-1)(i),(2,5,5-1)(i)$ and $(1,6,6-1)(i)$ in [2], as special cases.

The above considerations illustrate that by tabulating and closely inspecting formulae for mathematical functions, we can sometimes obtain new formulae.

In this note, we give another proof of the above theorem; Formula (1.5) can be easily derived by hand, and we realize that (1.5) closely relates to a strange evaluation by Gosper (see formula (2.5p).

\section{A Proof of Theorem 1}

In this section, we prove Theorem 1.

The following identity is easily verified:

$$
{ }_{2} F_{1}\left(\begin{array}{c}
\alpha, 1+\gamma \\
\gamma
\end{array} ; x\right)=\frac{(\alpha x-\gamma x+\gamma)(1-x)^{-\alpha-1}}{\gamma} .
$$

Now, we consider the case in which $\gamma$ approaches $-k$, where $k \in \mathbb{N}$. Then, the left-hand side of 2.1 becomes

$$
\begin{aligned}
& \sum_{n=0}^{k-1} \frac{(\alpha)_{n}(1-k)_{n}}{(-k)_{n} n !} x^{n} \\
& \quad-\frac{(\alpha)_{k+1}}{k(k+1) !} x^{k+1}\left[1+\frac{(\alpha+k+1)(2)}{(k+2) \cdot 1 !} x+\frac{(\alpha+k+1)_{2}(2)_{2}}{(k+2)_{2} \cdot 2 !} x^{2}+\cdots\right] \\
& ={ }_{2} F_{1}\left(\begin{array}{c}
\alpha, 1-k \\
-k
\end{array} ; x\right)-\frac{(\alpha)_{k+1}}{k(k+1) !} x^{k+1}{ }_{2} F_{1}\left(\begin{array}{c}
\alpha+k+1,2 \\
k+2
\end{array} ;\right) .
\end{aligned}
$$

Next, we evaluate

$$
{ }_{2} F_{1}\left(\begin{array}{c}
\alpha+k+1,2 \\
k+2
\end{array} ; \frac{k}{\alpha+k}\right) .
$$

From (40) in Section 2.8 of [4], we have

$$
\begin{aligned}
& {[c-2 b+(b-a) x]_{2} F_{1}\left(\begin{array}{c}
a, b \\
c
\end{array} ; x\right)} \\
& +b(1-x){ }_{2} F_{1}\left(\begin{array}{c}
a, b+1 \\
c
\end{array} ; x\right)-(c-b){ }_{2} F_{1}\left(\begin{array}{c}
a, b-1 \\
c
\end{array} ; x\right)=0 .
\end{aligned}
$$


Substituting $(a, b, c)=(\alpha+k+1,1, k+2)$ into $(2.3)$, this becomes

$$
[k-(\alpha+k) x]_{2} F_{1}\left(\begin{array}{c}
\alpha+k+1,1 \\
k+2
\end{array} ; x\right)+(1-x){ }_{2} F_{1}\left(\begin{array}{c}
\alpha+k+1,2 \\
k+2
\end{array} ; x\right)=(k+1) .
$$

This relation holds near $x=0$. Now, we carry out an analytic continuation of each side of (2.4) along a curve starting at $x=0$ and ending at $x=k /(\alpha+k)$. In this way, we obtain

$$
{ }_{2} F_{1}\left(\begin{array}{c}
\alpha+k+1,2 \\
k+2
\end{array} ; \frac{k}{\alpha+k}\right)=\frac{(\alpha+k)(k+1)}{\alpha} .
$$

This formula was first derived by Gosper in [7] (see also [2]).

Remark 2. As we now show, (2.5) holds for any $(\alpha, k)$ in the set

$$
\left\{(\alpha, k) \in \mathbb{C}^{2} ; \alpha \neq 0, \alpha+k \neq 0, k \neq-2,-3,-4, \ldots\right\} .
$$

First, we define

$$
F(x):={ }_{2} F_{1}\left(\begin{array}{c}
\alpha+k+1,2 \\
k+2
\end{array} ; x\right) .
$$

Then, because the radius of convergence of a non-terminating $F(x)$ is 1 , and because $F(x)$ is a multivalued function, we cannot uniquely determine its value for $x \in \mathbb{C}$ satisfying $|x| \geq 1$, in general. In other words, the value of $F(x)$ at such values of $x$ is ill-defined. However, as seen in the above, the value of $F(k /(\alpha+k))$ is uniquely determined and, hence, well-defined even if $|k /(\alpha+k)| \geq 1$.

From (2.1), (2.2) and (2.5), we find that

$$
\begin{aligned}
& \lim _{x \rightarrow k /(\alpha+k)} \lim _{\gamma \rightarrow-k}(\operatorname{lhs} \text { of }(2.1))={ }_{2} F_{1}\left(\begin{array}{c}
\alpha, 1-k \\
-k
\end{array} ; \frac{k}{\alpha+k}\right)-\frac{(\alpha+1)_{k}}{k !}\left(\frac{k}{\alpha+k}\right)^{k}, \\
& \lim _{x \rightarrow k /(\alpha+k)} \lim _{\gamma \rightarrow-k}(\operatorname{rhs} \text { of }(2.1))=0 .
\end{aligned}
$$

Thus, we have verified Theorem 1 except in the case $\alpha=0$. However, it is obvious that (1.5) holds when $\alpha=0$. This completes the proof of Theorem 1 .

\section{Acknowledgement}

This work is supported by a Grant-in-Aid for JSPS Fellows, JSPS No. 15J00201.

\section{References}

[1] A.Ebisu, On a strange evaluation of the hypergeometric series by Gosper, Ramanujan J. 32(2013), no. 1, 101-108. 
[2] A.Ebisu, Special values of the hypergoemetric series, Mem. Amer. Math. Soc., (to appear). also available e-Print arXiv:1308.5588.

[3] S.B.Ekhad, Forty "strange" computer-discovered[and computer-proved(of course)] hypergeometric series evaluations, The Personal Journal of Ekhad and Zeilberger, http://www .math.rutgers.edu/ zeilberg/mamarim/mamarimhtml/ strange.html, (2004).

[4] A.Erdélyi (editor), Higher transcendental functions Vol.1, McGraw-Hill, (1953).

[5] I.Gessel, Finding Identities with the WZ Method, J.Symbolic Computation, 20(1995), 537-566.

[6] I.Gessel and D.Stanton, Strange evaluations of hypergeometric series, SIAM J. Math. Anal., 13(1982), no. 2, 295-308.

[7] R.W.Gosper, A letter to D. Stanton, XEROX Palo Alto Research Center, 21 December 1977.

[8] W.Koepf, Hypergeometric summation - An algorithmic approach to summation and special function identities, Second edition, Universitext, Springer(2014).

[9] M.Petkovšek, H.Wilf and D.Zeilberger, $A=B$, A.K.Peters, Wellesley(1996).

[10] D.Zeilberger, Gauss's ${ }_{2} F_{1}(1)$ cannot be generalized to ${ }_{2} F_{1}(x)$, J. Comput. Appl. Math. 39(1992), no. 3, 379-382.

\author{
Akihito Ebisu \\ Department of Mathematics \\ Hokkaido University \\ Kita 10, Nishi 8, Kita-ku, Sapporo, 060-0810 \\ Japan \\ a-ebisu@math.sci.hokudai.ac.jp
}

